\title{
Automatic pigmented lesion segmentation through a dermoscopy- guided OCT approach for early diagnosis
}

Cristina L. Sarachaga, Sergio Lage, Maria Celia Morales, Ma Dolores Boyano, Aintzane Asumendi, et al.

Cristina L. Sarachaga, Sergio Lage, Maria Celia Morales, Ma Dolores Boyano, Aintzane Asumendi, Estibaliz Garrote, Olga M. Conde, "Automatic pigmented lesion segmentation through a dermoscopy-guided OCT approach for early diagnosis," Proc. SPIE 10851, Photonics in Dermatology and Plastic Surgery 2019, 108510K (26 February 2019); doi: 10.1117/12.2508002

SPIE. Event: SPIE BiOS, 2019, San Francisco, California, United States 


\title{
Automatic pigmented lesion segmentation through a dermoscopy- guided OCT approach for early diagnosis
}

\author{
Cristina L. Sarachaga*ab ${ }^{\text {ab }}$, Sergio Lage ${ }^{\mathrm{c}}$, Maria Celia Morales ${ }^{\mathrm{c}}, \mathrm{M}^{\mathrm{a}}$ Dolores Boyano ${ }^{\mathrm{c}}$, Aintzane \\ Asumendi ${ }^{\mathrm{c}}$, Estibaliz Garrote ${ }^{\mathrm{a}}$, Olga M.Conde ${ }^{\text {bde }}$ \\ ${ }^{a}$ Computer Vision Group, Smart Systems area, TECNALIA Research \& Innovation, Derio, Spain; \\ ${ }^{\mathrm{b}}$ Photonics Engineering Group, University of Cantabria, Santander, Spain; ${ }^{\mathrm{c} C e l l ~ B i o l o g y}$ and \\ Histology Department, University of the Basque Country (UPV-EHU), Leioa, Spain; ${ }^{\mathrm{d} C I B E R-B N N,}$ \\ Cantabria, Spain; ${ }^{\circledR}$ Instituto de Investigación Sanitaria Valdecilla (IDIVAL), Cantabria, Spain \\ *Cristina.lopez@tecnalia.com; phone +34 946430 850; tecnalia.com
}

\begin{abstract}
Early diagnosis of pigmented lesions, specially melanoma, is an unmet clinical need that would help to improve patient prognosis. Apart from histopathological biopsy, the only gold standard non-invasive imaging technique during diagnosis is dermatoscopy (DD). Over the last years, new medical imaging techniques are being developed and Optical Coherence Tomography (OCT) has demonstrated to be very helpful on dermatology. OCT is non-invasive and provides in-depth structural microscopic information of the skin in real-time. In comparison with other novel techniques, as Reflectance Confocal Microscopy (RCM), the acquisition time is lower and the field-of-view higher. Hence, consolidated diagnosis techniques and novel imaging modalities can be combined to improve decision making during diagnosis and treatment.

With actual methods, the delineation of lesion margins directly on OCT images during early stages of the disease is still really challenging and, at the same time, relevant from a prognosis perspective. This work proposes combining DD and OCT images to take advantage of their complementary information. The goal is to guide lesions delineation on OCT images considering the clinical features on DD images. The developed method applies image processing techniques to DD image to automatically segment the lesion; later, and after a calibration procedure, DD and OCT images become coregistered. In a final step the DD segmentation is transferred into the OCT image. Applying advanced image processing techniques and the proposed strategy of lesion delimitation, histopathological characteristics of the segmented lesion can be studied on OCT images afterwards. This proposal can lead to early, real-time and non-invasive diagnosis of pigmented lesions.
\end{abstract}

Keywords: Optical Coherence Tomography, OCT, dermatoscopy, image processing, image segmentation, diagnosis support

\section{INTRODUCTION}

Dermatologists require new non-invasive technologies for early diagnosis of skin cancer, especially for melanoma, since it becomes frequently confused with benign pigmented lesions. The current gold-standard imaging technique during diagnosis is dermatoscopy (Epiluminescence microscopy) [1]. In the latest years, advanced computer vision and machine learning methods have been successfully applied to dermatoscopic images, obtaining diagnostic results that are at the same level than expert dermatologists [2]. However, as the superficial information is not enough, the final diagnosis of the lesion is determined by the histopathological analysis through biopsy. In this sense, new imaging techniques can help improve patient prognosis if the diagnosis is made at early stages of the disease.

Reflectance Confocal Microscopy (RCM) [3] and Optical Coherence Tomography (OCT) [4] allow non-invasive, realtime, sub-surface inspection in an "optical-biopsy" like manner [5]. Portable devices of both technologies specially designed for dermatology are available on the market. RCM images are obtained horizontally (in the transversal plane) and OCT images are obtained axially in depth (coronal or sagittal plane) and 3D volumes of the lesion can be created. Although OCT images have less resolution than RCM images, the penetration capacity is higher and the acquisition time lower. Whereas the resolution of RCM devices varies from 1 to $3 \mu \mathrm{m}$ and the penetration capacity up to $200 \mu \mathrm{m}$, OCT

Photonics in Dermatology and Plastic Surgery 2019, edited by Bernard Choi,

Haishan Zeng, Proc. of SPIE Vol. 10851, 108510K · C 2019 SPIE

CCC code: $1605-7422 / 19 / \$ 18 \cdot$ doi: $10.1117 / 12.2508002$ 
devices can provide resolution less than $10 \mu \mathrm{m}$ with a penetration depth around $1.5 \mathrm{~mm}$ (up to $2 \mathrm{~mm}$ depending on the system). This OCT aspect is of great importance to evaluate the tumour invasion extension. OCT usefulness has been proven for clinical diagnosis and intervention (margin delimitation) of non-melanoma skin cancer in cases of Basal Cell Carcinoma (BCC) [6][7][8], but its use in the diagnosis of melanocytic pigmented lesions (melanoma vs nevus) is still under discussion [9][7][10].

OCT image interpretation is still a challenge for clinicians and the required learning curve slows down the adoption of OCT on the dermatological clinical routine. The constraints in the analysis of OCT data is due to its double nature: photonics and image. This makes necessary to jointly apply signal processing techniques to deal with the noisy nature of the signal [11][12] and image processing techniques to exploit the spatial correlation of the biological structures. Image processing techniques can be applied with the goal of performing automatic interpretation of the images for delimitation or classification tasks, and hence facilitate the acceptance of the technology. However, due to the mentioned challenges, there have been just few attempts of applying these kinds of methods to OCT images of the skin [13][14][15] on tasks that aim automatic classification. The results obtained so far do not have enough sensibility and specificity, and thus cannot be adopted in the clinical routine. To some extent, the reason for this is that OCT is still on the early steps of application on dermatology and not enough data for automatic classification is available.

In the short term, the best solution to facilitate the adoption of new imaging techniques could be the combination with well-established techniques in the field on application. For this reason, this work proposes combining OCT and dermatoscopy on the diagnosis of dermatological lesions. Although the automatic processing of OCT images is a very powerful solution, the clinicians have the feeling of losing control over the interpretation of the images during diagnosis. In this regard, OCT imaging can be introduced progressively in the clinical routine. For instance, assisting lesion delimitation with dermatoscopy, so the clinician only has to focus on the identification of clinical features within the margins of the lesion. Then, lesion diagnosis can be progressively mastered, and the adoption of the technology naturally accomplished. This purpose can be achieved with a method that implements the following steps: 1) to apply image processing techniques to DD image to automatically segment the lesion; 2) to co-register DD and OCT images after a calibration procedure; 3 ) to transfer the DD segmentation into the OCT image.

Lesion segmentation over dermatoscopy images is still an active issue that has been strongly investigated in computer vision since the 90s [16]. Image processing challenges as the "Skin Lesion Analysis Towards Melanoma Detection" sponsored by the International Skin Imaging Collaboration (ISIC) organization [17] have promoted its analysis even more. Traditional approaches adopt both image processing and machine learning techniques, as for example: thresholding, clustering, edge operators, regional segmentation, morphological operators, active contours, fuzzy logic, supervised learning, etc [18].

The development of the deep learning paradigm has played a crucial role in dermatoscopy lesion segmentation and automatic classification in the past few years. Unprecedent performance results have been obtained with this kind of techniques, obtaining sensibility and specificity rates that outperform expert dermatologists [2][19]. As it can be observed in the ISIC challenge leader board of the past two years [20][21], the winning strategies are dominated by deep learning. However, there are still some challenges to solve on the commercialization of computer aided diagnosis software (CAD) including this type of algorithms, as improving the diagnosis of atypical melanoma lesions [22], where the number of samples is scarce and its usefulness in the clinical routine very important.

Independently from the type of method used for lesion segmentation, image pre-processing strategies have demonstrated to be necessary for improving the performance of the delimitation algorithm. One common problem is the elimination of artifacts, as bubbles, vessels, color charts, ruler marks, dark corners, ink, thin/thick hair, etc. [23]. Another common problem is the variability of color and illumination or the lack of contrast in the images of the database. Color normalization strategies are required and its usefulness has been proven [24].

When applying machine learning strategies for classification purposes and developing CAD software, prior segmentation of the target object is required as a basic step that has demonstrated to improve the results [25][26]. As proposed in this work, this task can be accomplished over OCT images using dermatoscopy images of the same lesion as a reference. Then, automatic lesion classification can be performed only over OCT images, or also combining the classification results of OCT images and dermatoscopy images. Due to the complementary nature of both imaging modalities, this latest approach results more interesting from the clinical point of view. A solution of these characteristics can lead to achieving real-time and non-invasive diagnosis of dermatological lesions. 


\section{MATERIALS AND METHODS}

Being dermatoscopy the most common tool in the clinical routine, this work proposes taking advantage of the information and features that can be found on this image and transfer them into the corresponding OCT image of the same lesion. Then, lesion histopathological features on OCT images can be studied with more confidence and clinicians get used progressively to this new diagnosis imaging modality.

This proposal can be achieved adopting image processing and machine learning techniques. Different algorithms and strategies can be implemented at each step, and mostly depend on the technical characteristics of the images and the OCT device being used [27]. Whether the device has been organically designed for dermatological purposes or not makes the difference. For example, VivoSight ${ }^{\circledR}$ (Michelson Diagnostics) [28] provides dermatoscopy and OCT images of the area of analysis at the same time, which facilitates co-registration of images. The NITID device (Medlumics) [29] used in this study also provides this feature, and the scanning area with the OCT is clearly marked on the dermatoscopy image. Other devices as Callisto ${ }^{\circledR}$ (Thorlabs) [30] do not provide even this functionality and the dermatoscopy images have to be captured with an external device, which requires more effort in calibration. Unfortunately, none of these systems provide dermatoscopy delimitation transfer for OCT segmentation.

\subsection{Imaging equipment}

Images used in this work have been acquired using the NITID device developed by Medlumics [31]. The device provides clinical, dermatoscopy (14 mm, $1.16 \mathrm{mpx}$ and 20-40x magnification, $1920 \mathrm{x} 1080$ pixel) and OCT images (selectable FOV from 3 to $12 \mathrm{~mm}, 11 \mu \mathrm{m}$ axial resolution and $12 \mu \mathrm{m}$ lateral resolution, 425-1700 x 512 pixels) for each lesion analyzed. The dermatoscopy images contain a white line in the middle indicating the approximate scanning path on the OCT image (B-scan). Figure 1 shows an example of a nevus lesion, with a field-of-view (FOV) of $6 \mathrm{~mm}$ on the OCT image.

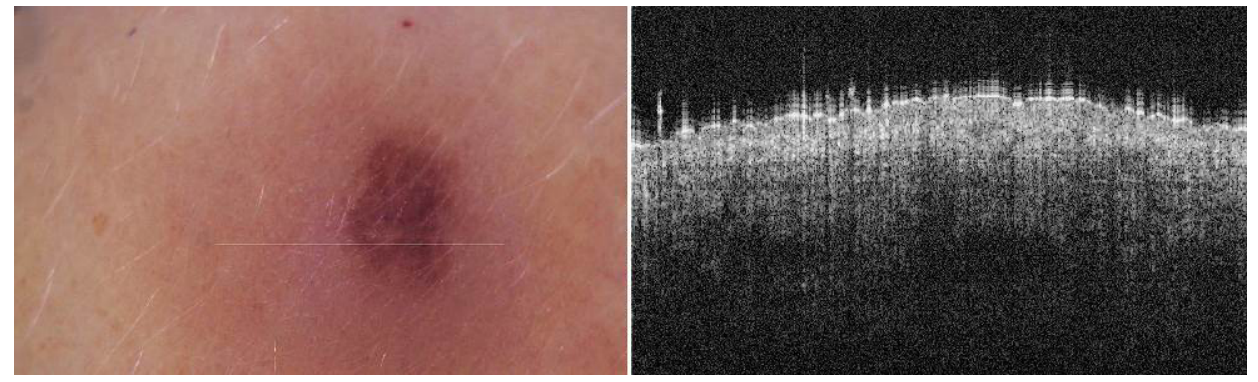

Figure 1. Example dermatoscopy and OCT image from the equipment

\subsection{Skin Lesions Database}

The private database contains images (clinical, dermatoscopy and OCT) and clinical data of real patients from the Cruces University Hospital and Basurto University Hospital (Spain). All the data has been acquired with the approval of the Euskadi Clinical Research Ethics Committee (CEIC) and the hospitals' ethical committees. Dermatologist diagnosis, histopathological diagnosis and histopathological features have been also collected for each lesion included in the database.

Experiments have been performed with a database collected during five months at the hospitals that contains: 62 melanomas, 76 nevi and 124 healthy lesions. More samples were collected during this period, though they had to be discarded due to the presence of severe artifacts on the OCT images that make them unusable for diagnosis. Most of encountered artifacts are caused by dark and thick hair or the presence of blood on ulcerated lesions. For example, thick hair affects the acquisition of the OCT image because it blocks the penetration of light into the tissue and in some cases makes impossible the evaluation of the lesion with OCT. On the other side, in the case of ulceration, the epidermis and dermis of the area is affected and hence its properties cannot be studied, although the healing scab is visible on the OCT image. See Figure 2 for an example of the mentioned artifacts. Figure 2(a) displays the effect of hair and Figure 2(b) the presence of ulcerations and blood. 

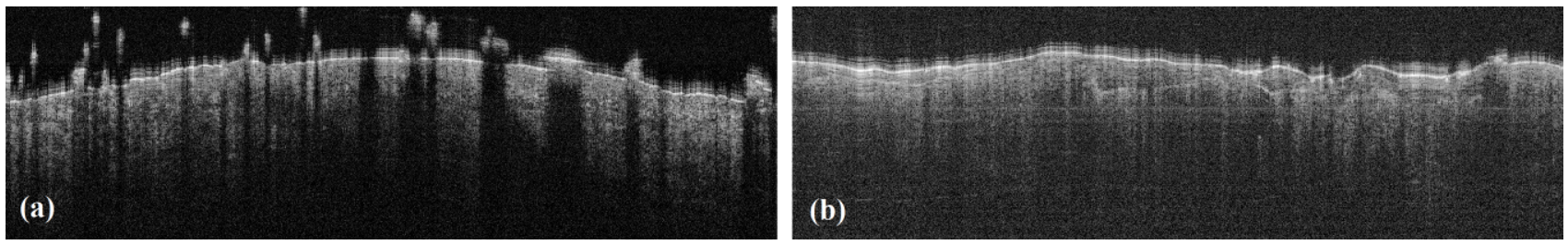

Figure 2. Examples of artifacts on OCT images. (a): thick dark hair; (b): ulceration and blood

\subsection{Methodology}

A simple approach using image processing methods has been implemented in Python to demonstrate the feasibility and utility of the proposed methodology. The different steps are explained in detail and results obtained at each step included on section 3 .

\section{Lesion segmentation}

The automatic segmentation methods reviewed at the introductory section, consider that the image analysis is performed over the whole dermatoscopy image or in a region-of-interest (ROI) area surrounding the entire lesion [18]. In that sense, a proposal was presented at ISIC 2017, achieving the $8^{\text {th }}$ position in lesion segmentation [32]. This proposal, that includes a pre-processing strategy for color compensation and a deep learning method for lesion segmentation have been discarded in this implementation for its complexity and the reasons presented below.

Considering that OCT B-scans only provide local in-depth information of the tissue, overall segmentation of the lesion on the dermatoscopy image is not required. Therefore, the proposed method recommends focusing the segmentation on extracting the local characteristics of the lesion on the area where the OCT is acquired. This has been done defining a region-of-interest (ROI) area surrounding the OCT scanning path, as shown by the blue box area marked in Figure 3(b). This process has required to perform two steps: 1) automatic detection of the white line (OCT scanning path) on the dermatoscopy image and 2) to calculate the surrounding ROI. The white line has been automatically detected by calculating a binary mask using the value 255 (white) as threshold first, secondly applying a morphological operation to remove small objects (to eliminate glare and reflections that occasionally appear on dermatoscopy images) and finally computing the beginning and ending pixels of the line on the final mask. After this, an area of 10 pixels before and after the calculated line path has been defined as the surrounding ROI area. This approach is only feasible when the OCT device being used provides both dermatoscopy and OCT images, so the OCT scanning path can be determined. An OCT device without dermatoscopy would require adopting an alternative and possibly more complex calibration strategy for determining the scanning area.
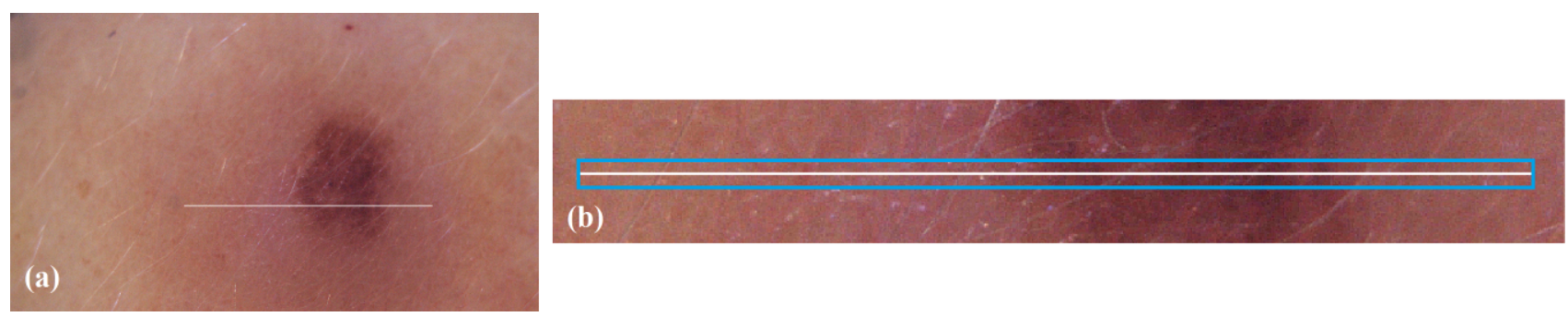

Figure 3. (a): Dermatoscopy image with OCT scanning path (white line), (b): ROI area of study of local features of dermatoscopy image for lesion segmentation (blue box).

Having identified the ROI on the dermatoscopy image, the lesion delimitation can be performed with conventional solutions. Two different strategies have been implemented and compared in this step: Otsu thresholds [33] and gradients [34]. The Otsu strategy comprises the following steps: 1) to calculate the Otsu threshold within the sub-region; 2) to generate preliminary segmentation obtaining a binary mask with the threshold value; 3) to smooth the binary mask applying median filter (disk=3); 4) to refine the segmentation removing small objects (ex. thin dark hair) within the mask (th=10) and 5) to calculate the left and right limits (indexes) from segmentation mask. On the other side, gradients have been studied as follows: 1) to calculate 1d-array with the mean values of the columns within the sub-region; 2) to apply a rough median filter to smooth the $1 \mathrm{~d}$ signal (kernel=99) and to eliminate small changes (ex. Hairs); 3 ) to calculate the nth derivatives of the signal; 4) to automatically detect the biggest changes (peaks) on the signal and 5) to assign the first 
and last peak detected on the signal as delimitation limits. The sub-regions before and after the white line (Figure 3(a)) have been studied independently with each strategy and the results merged afterwards. The before and after threshold values calculated are compared and the minimum and maximum values are determined as the limits of the lesion. A comparison of the results obtained with each strategy is illustrated on Figure 9.

The implemented strategy has taken into consideration that small artifacts as thin hair are present on the lesions and affect lesion delimitation on the dermatoscopy images. In the presence of thick dark hair, it can be necessary to apply some pre-processing to get rid of them [35][36] and avoid errors in the segmentation. This operation will be only useful if the associated OCT image is not very much affected by the artifacts caused by hair and still valid for diagnosis, as the example shown on Figure 4. In some cases, these kinds of artifacts make impossible the evaluation of the lesion with OCT as in Figure 2(a).
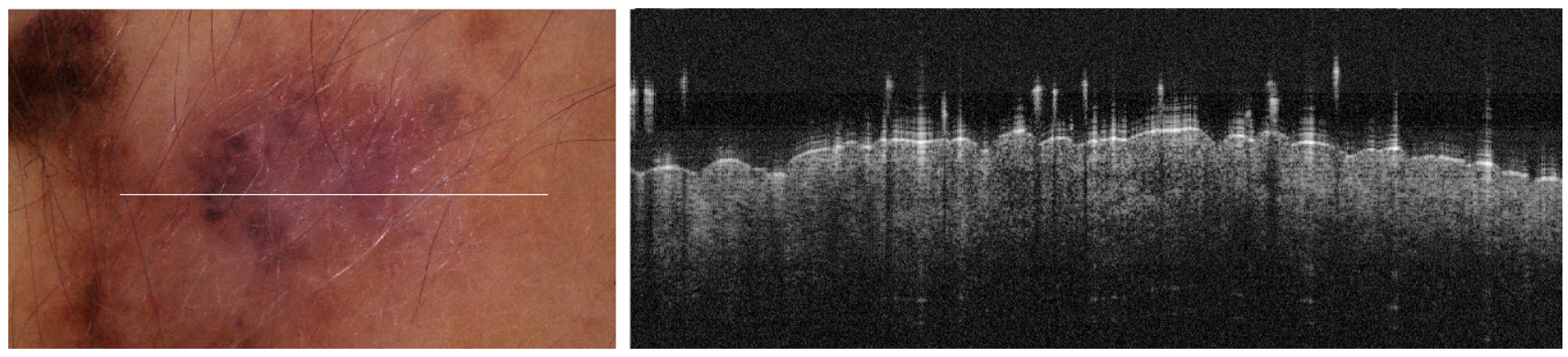

Figure 4. Effect of artifacts caused by thin hair

\section{Co-registration}

Co-registration of the dermatoscopy and OCT images is an important step, as it allows to compare both images in the same scale and coordinates system. This process is reasonably viable when the device provides both dermatoscopy and OCT images at the same time of scanning the lesion. It mainly requires performing land-marking and device calibration operations before image co-registration. If the OCT devices does not provide dermatoscopy images, it can also be pursued, but the land-marking process will be much more complex.

The device employed in this work provides an estimation of the OCT scanning path on dermatoscopy images (Figure 1), but if this feature is not available or needs confirmation, external landmarks can be employed. The usage of land-marks has already been explored by some clinicians very active on the OCT dermatoscopy community [37]. Its usefulness on the clinical practice before BCC surgery has been successfully demonstrated [38]. Inspired by this work, experiments with two different types of markers have been performed: 1) silver metallic pen (Pentel ${ }^{\mathbb{B}}$, Hybrid Gel Grip Metallic) and 2) black permanent marker (Staedtler Lumocolor ${ }^{\circledR}$ permanent). Results obtained are illustrated on Figure 5 and Figure 6 where the area within the landmark limits is indicated in blue on the OCT images. Whereas the silver metallic pen mark blocks the penetration of light into the tissue due to the effect of reflection, the black pen mark is not opaque enough to block the light, and only some surface eco minimization is observed. Various experiments were performed with both markers, and the results obtained have been always the same, determining that the usage of a silver metallic pen is the most favorable strategy for lesion land-marking. Besides, it's a common practice that tumor resection margins are marked on the skin with a permanent pen prior the surgical procedure, so this procedure is not unknown for clinicians. Other strategies, as for example using stickers, should be discarded because the glue can cause allergic reactions on the skin on the patient.

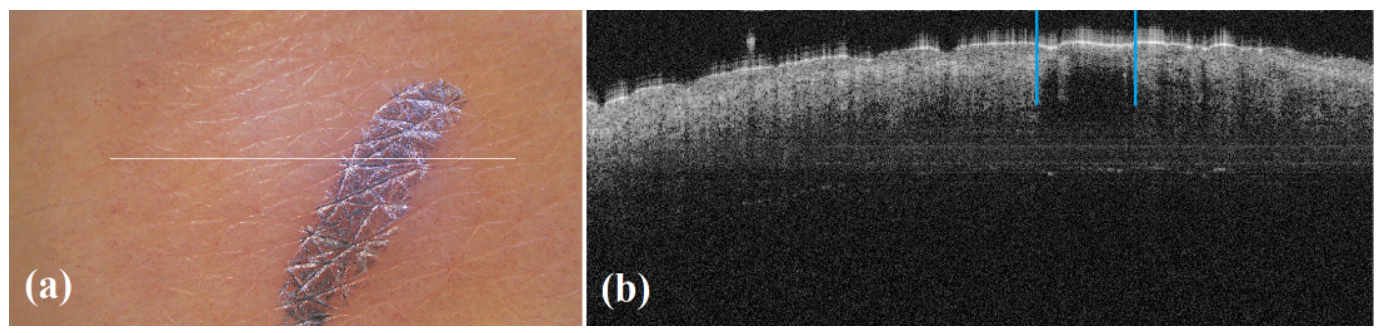

Figure 5. Marking example using silver metallic pen. (a): dermatoscopy image of healthy skin with silver metallic pen landmark; (b): OCT image with land-mark area highlighted in blue. 


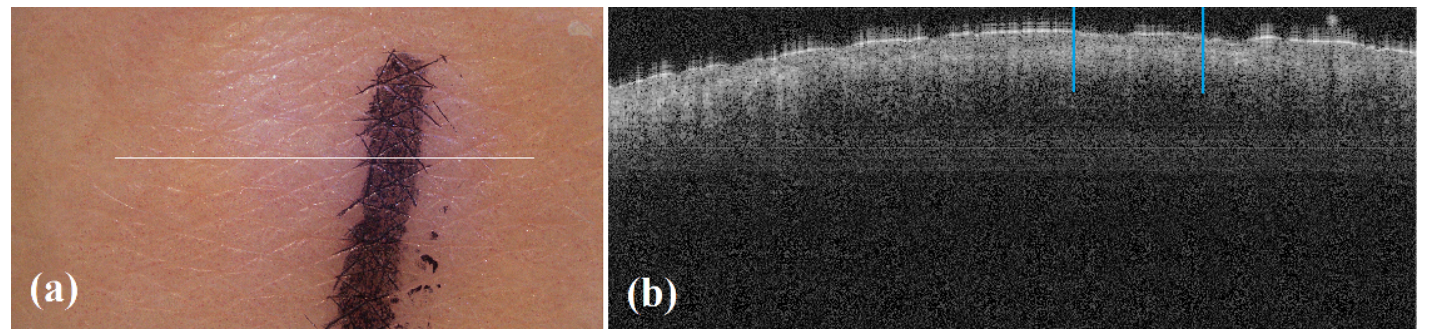

Figure 6. Marking example using black permanent pen. (a): dermatoscopy image of healthy skin with black permanent pen land-mark; (b): OCT image with land-mark area highlighted in blue.

Once the scanning path has been confirmed via landmarks, it's important to perform some calibration operations in order measure the real FOV of the OCT images. For this purpose, if available, standard calibration cards can be used (USAF 1951, NBS 1952, etc.). In this work, the calibration has been performed using an ordinary element, a barcode. Figure 7 shows two acquisitions where deviation on the estimated scanning path (edited as red line on dermatoscopy images to add contrast) with respect to the features on the OCT image can be observed. The $9 \mathrm{~mm}$ FOV example (a)(b) demonstrates that the beginning of the path is not properly established and needs to be adjusted. Besides, the $12 \mathrm{~mm}$ FOV example (c)(d) reveals that the lines present are the beginning and end of the path are not visible on the OCT image. Using these and additional examples and $\mathrm{px}$ to $\mathrm{mm}$ ratio provided by the device manufacturer, the deviation observed has been calculated. For example, for the $9 \mathrm{~mm}$ FOV case, approximately $75 \mathrm{px}(0,5 \mathrm{~mm})$ at the beginning of the scanning path on the dermatoscopy ROI image must be deleted to achieve a correct image co-registration. These calculations have been made for all the FOV measures provided by the device in order to automatically apply the required corrections in all the database images.

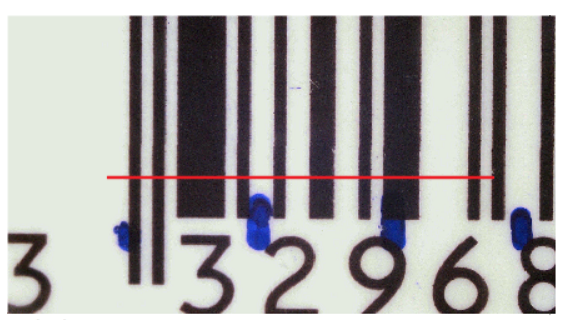

(a)

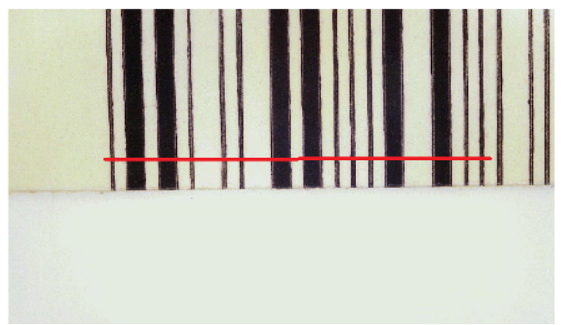

(c)

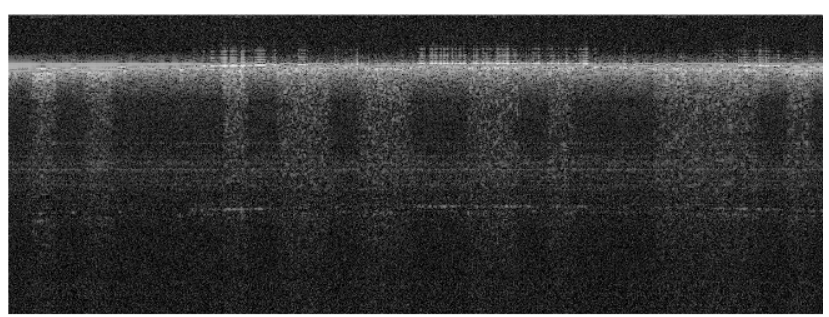

(b)

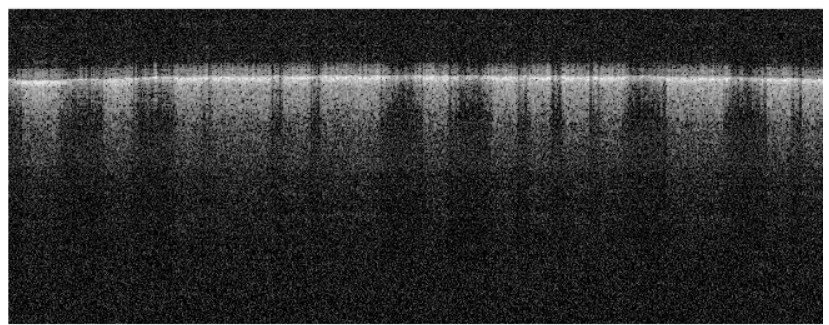

(d)

Figure 7. Example of calibration using a barcode. (a)(c): Dermatoscopy images with estimated OCT scanning path marked on red; Right: OCT image where the bar codes are visible. (b)(d): 9mm FOV comparison, Bottom: 12mm FOV comparison.

An example of a correct co-registration operation is shown at Figure 8, where the deviation on the scanning path at the dermatoscopy image is visible. Once the necessary corrections have been calculated, the co-registration of the images can be completed. First, a sub-image of the dermatoscopy image has been extracted, following the same procedure applied before for the extraction of the ROI region. However, in this case the while line has to be corrected with the values calculated on the calibration. This has been done deleting 75 pixels at the beginning of the ROI area that are not visible on the OCT image. Second, both the dermatoscopy ROI and the OCT images have been concatenated for 
comparison (using a vertical stack image operation), as they are on the same scale after all the operations previously performed at this step. Figure 9 exemplifies the result of the co-registration operation.

\section{Segmentation transfer}

This last step unifies the results obtained in the previous phases: the lesion segmentation performed on the dermatoscopy image and the co-registered values after calibration. The delimitation step, provided lateral delimitation values (left and right) of the lesion. These values indicate two column numbers with respect to ROI area calculated for the analysis (Figure 3). After the co-registration operation, and to translate them to the OCT image, the delimitation values must be corrected. The deviation values calculated during the calibration denote the correction to be applied. For example, in this work, for an image acquired with 9mm FOV, 75px at the beginning of ROI area must be discarded. Then, the final OCT lateral delimitation delimitating values can be corrected by simply subtracting $75 \mathrm{px}$ to initial delimitating values. The results of this last step are depicted in Figure 11.

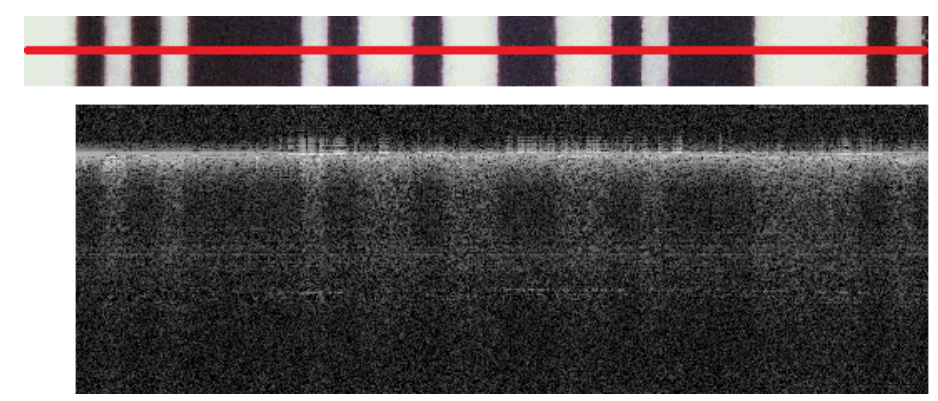

Figure 8: Example of correct co-registration. Scanning path deviation detected and needs correction.

\section{RESULTS AND DISCUSSION}

The methodology proposed in section 2.3 has been tested with the database images acquired using the equipment mentioned in section 0. Images illustrating the results obtained at each step are included below for the analysis of the results.

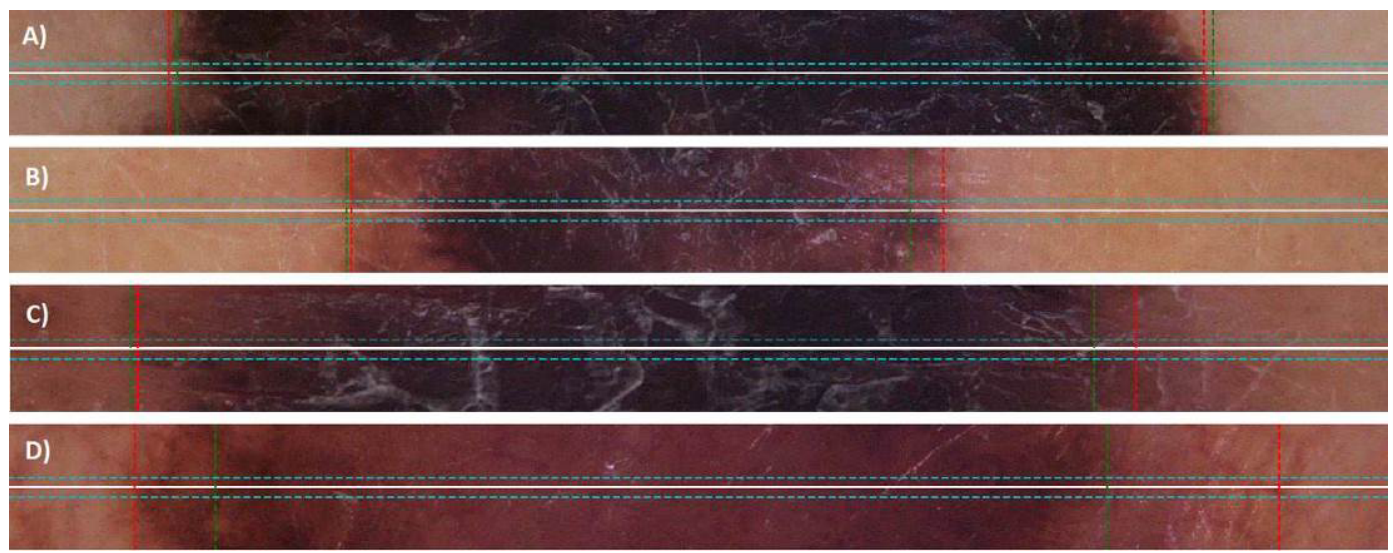

Figure 9. Local lesion delimitation (using ROI area in blue box) on melanoma lesions (A, B) and nevus lesions (C, D). Legend: red lines are Otsu thresholding segmentation; green lines are slope derivatives segmentation.

The first step of the methodology is lesion delimitation, where two different methods have been implemented for comparison: Otsu thresholds and gradients. Figure 9 includes various examples where the results of both methods are illustrated. The blue box indicates the ROI defined for the local analysis of lesion delimitations. The outcome of the Otsu filter is depicted with red vertical lines, whereas the outcome of the gradients derivatives is depicted with green vertical lines. As illustrated on the examples included below, the Otsu strategy seems to adjust better to the limits of the lesions according to the dermatoscopy image. This tendency is observed in all the images of the database that have been processed. For this reason, Otsu threshold has been selected as the reference 
delimitation method on the implemented methodology. Furthermore, Figure 9 results demonstrate that a simple strategy can be adopted for lesion delimitation when analyzing local features on a dermatoscopy image.

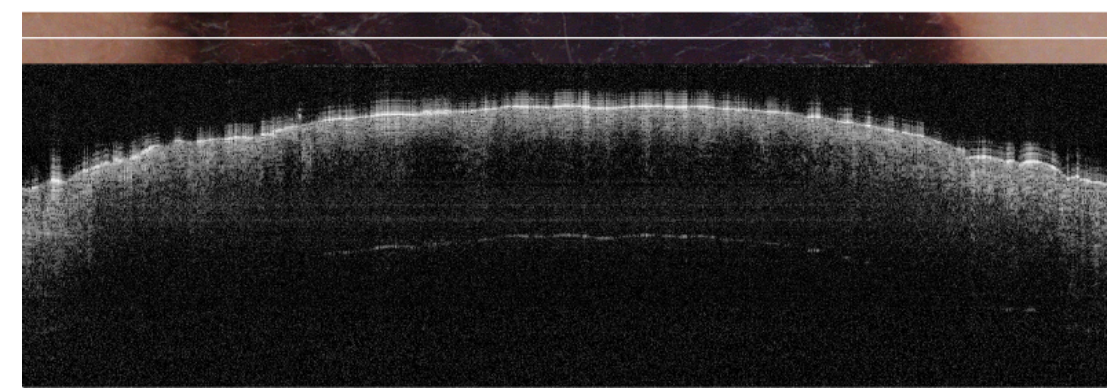

Figure 10. Co-registration operation after OCT device calibration

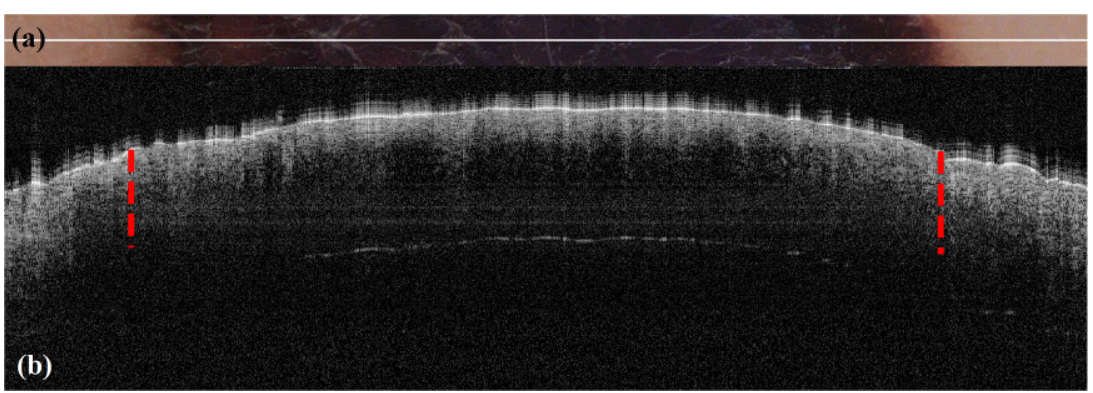

Figure 11. Segmentation transfer example. (a): Dermatoscopy image, (b): OCT images with delimitation landmarks

The co-registration performed as second step of the methodology transforms the dermatoscopy and OCT images to the same scale, which makes them comparable. The results obtained at this step are illustrated with one example on Figure 10. The dermatoscopy and OCT images are concatenated for comparison. The co-registration of both images modalities is required to be able to perform the last step of the methodology: segmentation transfer.

The results of the segmentation transfer step are depicted at Figure 11. The horizontal dotted red lines represent the lateral delimitation of the lesion obtained with the Otsu strategy at the first step of the methodology. The images generated at this last step can be included in a CAD software for assisting the clinicians on OCT image interpretation and hence smooth the learning curve of the technology. Including visual aids over the OCT images (as the red marks show in the example) can help them to focus their attention on analyzing the structural and histological features inside the lesion with respect to the healthy surrounding tissue.

The lateral delimitation presented in this work can be complemented with upper (air-tissue) and lower (besides dermis) delimitation for a complete segmentation of the lesion on the OCT image. These additional delimitations are out of the scope of this work, and hence not explained here. Detailed examples including the overall delimitation of the lesion on the OCT images are presented below. Figure 12 includes two examples of nevus lesions and Figure 13 two examples of melanoma lesions. The compound nevus example can be further studied at Figure 14. This figure includes the Hematoxilin-Eosine (HE) biopsy scanned image with the goal to compare the histopathological features within the margins of the lesion on the OCT image. Several images like this have been analyzed into detail at the Basurto University hospital (Spain) with the support of the pathologists. In this example, the HE image reveals the presence of groups of nevus cells (mature melanocytes) in the dermis of the lesion. This characteristic can also be observed within the lesion delimitation margins calculated for the lesion on the OCT, as indicated by the blue arrows on the image. An additional example of a spreading melanoma case is illustrated at Figure 15. The tumoral nests identified by the pathologist at the HE image can be also identified at the OCT image within the calculated margins, as indicated with the arrows on both images. 

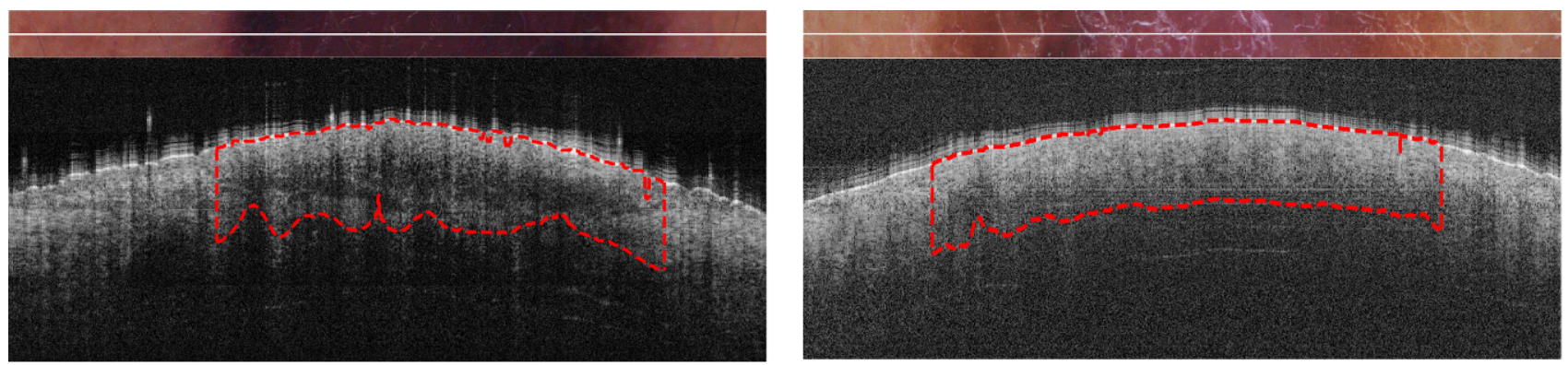

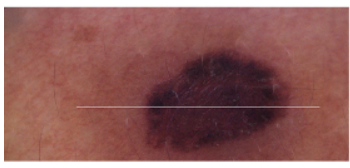

(a)
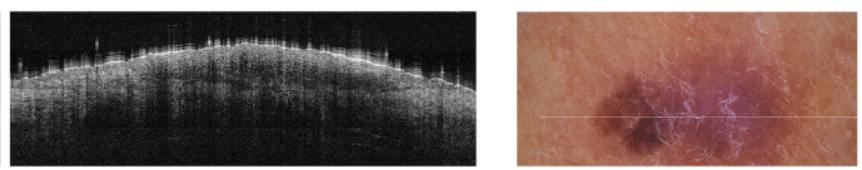

(b)

Figure 12. Methodology results with nevus examples. (a): Compound nevus, (b): Lentiginous nevus

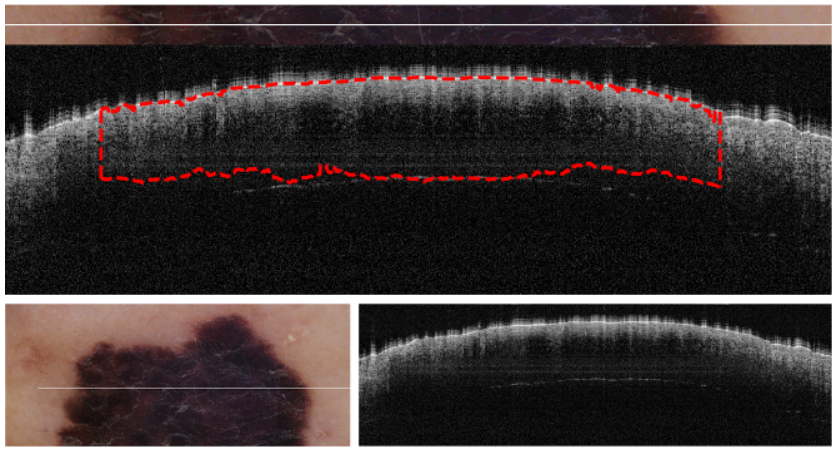

(a)

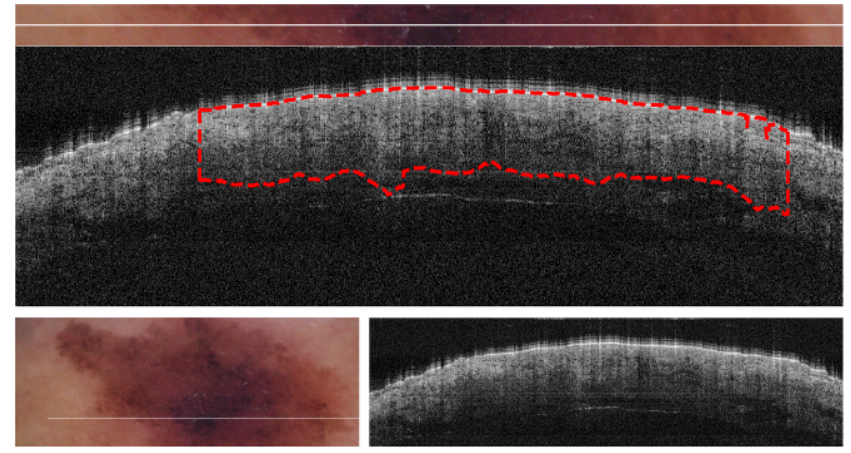

(b)

Figure 13. Methodology results with melanoma examples. (a): Melanoma In-situ, (b): Superficial spreading Melanoma

The overall approach of lesion delimitation on OCT images assisted by dermatoscopy presented in this work is particularly helpful when the goal is to apply machine learning methods for automatic classification of lesions over OCT images. As mentioned before, the prior segmentation of the lesion is required so characteristics of the lesions can be studied for classification. In the latest years, advanced methods based on the deep learning paradigm are gaining all the attention in the machine learning world. Deep learning strategies over OCT images are being applied with success on ophthalmology for automated lesion classification [39][40]. No references on dermatology have been found at the time of writing this work. 

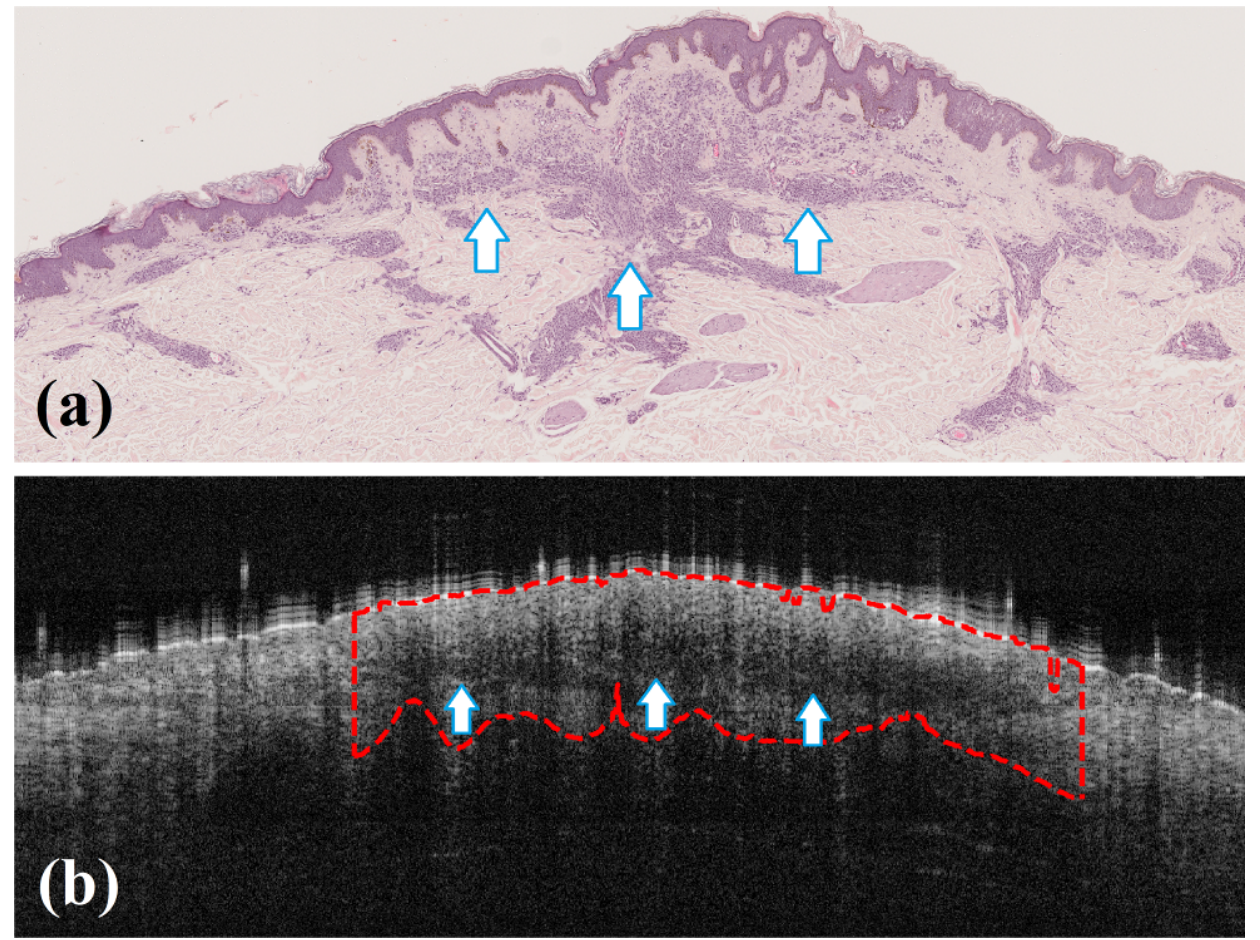

Figure 14. Study of histopathological features on OCT image after lesion segmentation on a Compound Nevus sample. Arrows in both images indicating the presence of groups of nevus cells. (a): HE biopsy; (b): OCT image including lesion segmentation.
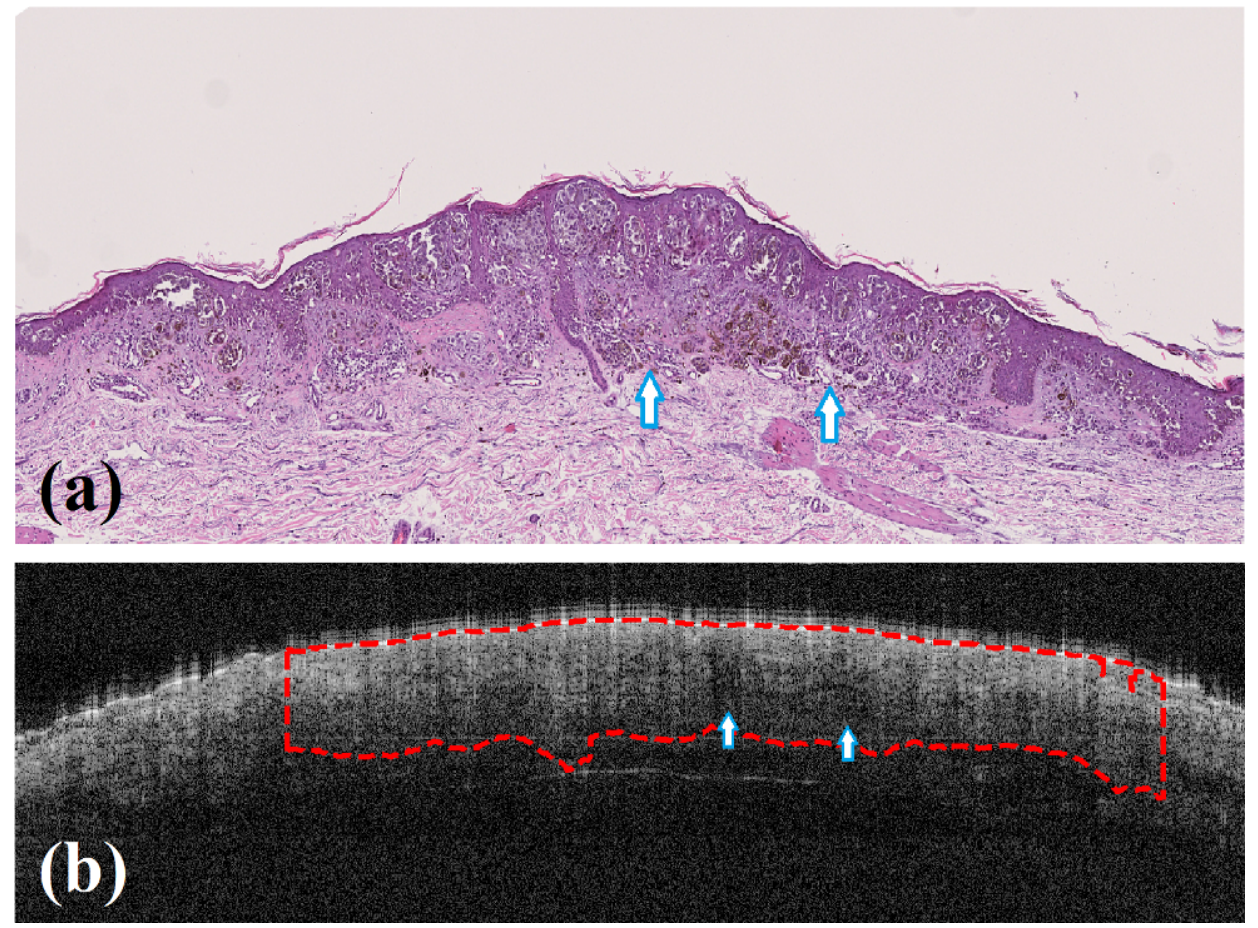

Figure 15. Study of histopathological features on OCT image after lesion segmentation on a Superficial spreading Melanoma sample. Arrow in both images indicating the presence of tumoral nests. (a): HE biopsy; (b): OCT image including lesion segmentation. 


\section{CONCLUSIONS}

The real-time correct diagnosis of dermatological lesions at the early stages of the diseases is extremely challenging with current diagnosis methods. Improving the differentiation of benign (ex. nevus) and malign (ex. melanoma) pigmented lesions is highly demanded by clinicians. In that sense, new imaging technologies, as Optical Coherence Tomography (OCT), allow to study the internal histopathological features of the lesion in real-time. However, the interpretation of OCT images poses a challenge for clinicians and prevents them from adopting the technology.

This work has proposed combining a well-established imaging technology as Epilluminescence microscopy (dermatoscopy) with OCT. The goal of the combination has been to automatically perform lateral lesion delimitation over OCT images with the information present on dermatoscopy images. This has required to implement three different steps: 1) lesion delimitation of dermatoscopy image, 2) dermatoscopy and OCT images co-registration and 3) segmentation transfer to OCT image. The results presented reveal that this strategy can be successfully implemented.

The automated delimitated strategy proposed could be included in a Computer Aided-Diagnosis (CAD) software to assist the clinician during diagnosis. The estimated lesion delimitation over the OCT images can be indicated with visual aids so the clinicians can focus on interpretating the clinical features within the lesion. Additionally, machine learning classification methods can be applied after the segmentation and results included into the CAD software to further assist the clinicians on the real-time diagnosis of the lesions.

\section{ACKNOWLEDGEMENTS}

This work has been developed thanks to the funding of the ECSEL European project ASTONISH (ID.692470) and Basque Country (Spain) ELKARTEK projects MELAMICS (KK-2016-00036) and MELAMICS II (KK-2017/00041). Special thanks to the dermatologists and personnel of the Cruces University Hospital (Cruces, Spain) and the Basurto University Hospital (Bilbao, Spain) for their collaboration on the generation of the annotated database from real patients.

\section{REFERENCES}

[1] Binder, M., Schwarz, M., Winkler, A., Steiner, A., Kaider, A., Wolff, K., and Pehamberger, H., "Epiluminescence microscopy. A useful tool for the diagnosis of pigmented skin lesions for formally trained dermatologists.," Archives of dermatology 131(3), 286-91 (1995).

[2] Esteva, A., Kuprel, B., Novoa, R.A., Ko, J., Swetter, S.M., Blau, H.M., and Thrun, S., "Dermatologist-level classification of skin cancer with deep neural networks," Nature 542(7639), 115-118 (2017).

[3] Levine, A., and Markowitz, O., "Introduction to reflectance confocal microscopy and its use in clinical practice.," JAAD case reports 4(10), 1014-1023 (2018).

[4] Fujimoto, J., and Swanson, E., "The Development, Commercialization, and Impact of Optical Coherence Tomography," Investigative Ophthalmology \& Visual Science 57(9), OCT1-OCT13 (2016).

[5] Welzel, J., and Schuh, S., "Noninvasive diagnosis in dermatology," JDDG: Journal der Deutschen Dermatologischen Gesellschaft 15(10), 999-1016 (2017).

[6] Holmes, J., and Welzel, J., [OCT in Dermatology], in Opt. Coherence Tomogr. Technol. Appl. 2nd Ed., Springer International Publishing, Cham, 2189-2207 (2015).

[7] Olsen, J., Holmes, J., and Jemec, G.B.E., "Advances in optical coherence tomography in dermatology-a review," Journal of Biomedical Optics 23(04), 1 (2018).

[8] Schuh, S., Kaestle, R., Sattler, E.C., and Welzel, J., "Optical coherence tomography of actinic keratoses and basal cell carcinomas - differentiation by quantification of signal intensity and layer thickness," Journal of the European Academy of Dermatology and Venereology 30(8), 1321-1326 (2016).

[9] Levine, A., Wang, K., and Markowitz, O., "Optical Coherence Tomography in the Diagnosis of Skin Cancer," Dermatologic Clinics 35(4), 465-488 (2017).

[10] Gambichler, T., Pljakic, A., and Schmitz, L., "Recent advances in clinical application of optical coherence tomography of human skin," Clinical, Cosmetic and Investigational Dermatology 8, 345-54 (2015).

[11] Freund, I., "Joseph W. Goodman: Speckle Phenomena in Optics: Theory and Applications," Journal of Statistical Physics 130(2), 413-414 (2007). 
[12] Fercher, A.F., [Inverse Scattering, Dispersion, and Speckle in Optical Coherence Tomography], in Opt. Coherence Tomogr. Technol. Appl., W. Drexler and J. G. Fujimoto, Eds., Springer Berlin Heidelberg, Berlin, Heidelberg, 119-146 (2008).

[13] Raupov, D.S., Myakinin, O.O., Bratchenko, I.A., Zakharov, V.P., and Khramov, A.G., "Skin cancer texture analysis of OCT images based on Haralick, fractal dimension, Markov random field features, and the complex directional field features," in Proc. SPIE 9887, J. Popp, V. V. Tuchin, D. L. Matthews, and F. S. Pavone, Eds., 100244I (2016).

[14] Adabi, S., Conforto, S., Hosseinzadeh, M., Noe, S., Daveluy, S., Mehregan, D., and Nasiriavanaki, M., "Textural analysis of optical coherence tomography skin images: quantitative differentiation between healthy and cancerous tissues," 2017, 100533F.

[15] Boone, M.A.L.M., Suppa, M., Marneffe, A., Miyamoto, M., Jemec, G.B.E., and Del Marmol, V., “A new algorithm for the discrimination of actinic keratosis from normal skin and squamous cell carcinoma based on in vivo analysis of optical properties by high-definition optical coherence tomography," Journal of the European Academy of Dermatology and Venereology 30(10), 1714-1725 (2016).

[16] Celebi, M.E., Mendonca, T., and Marques, J.S., [Dermoscopy image analysis], CRC Press (2015).

[17] "ISIC 2018 | ISIC 2018: Skin Lesion Analysis Towards Melanoma Detection," Int. Ski. Imaging Collab., 2018, $<$ https://challenge2018.isic-archive.com/> (10 December 2018).

[18] Celebi, M., Wen, Q., Iyatomi, H., Shimizu, K., Zhou, H., and Schaefer, G., [A State-of-the-Art Survey on Lesion Border Detection in Dermoscopy Images] , 97-129 (2015).

[19] Haenssle, H.A., Fink, C., Schneiderbauer, R., Toberer, F., Buhl, T., Blum, A., Kalloo, A., Hassen, A.B.H., Thomas, L., et al., "Man against machine: diagnostic performance of a deep learning convolutional neural network for dermoscopic melanoma recognition in comparison to 58 dermatologists," Annals of Oncology (2018).

[20] "Leaderboards | ISIC 2017: Skin Lesion Analysis Towards Melanoma DetectionPart 1: Lesion Segmentation," 2017, <https://challenge.kitware.com/\#phase/584b0afacad3a51cc66c8e24> (14 December 2018).

[21] "Leaderboards | ISIC 2018," <https://challenge2018.isic-archive.com/leaderboards/> (14 December 2018).

[22] Mar, V.J., and Soyer, H.P., "Artificial intelligence for melanoma diagnosis: how can we deliver on the promise?," Annals of Oncology 29(8), 1625-1628 (2018).

[23] Mishra, N.K., and Celebi, M.E., "An Overview of Melanoma Detection in Dermoscopy Images Using Image Processing and Machine Learning" (2016).

[24] Fidalgo Barata, A., Celebi, E., and Marques, J., "Improving Dermoscopy Image Classification Using Color Constancy," IEEE Journal of Biomedical and Health Informatics1-1 (2014).

[25] Møllersen, K., Zortea, M., Hindberg, K., Schopf, T., Skrøvseth, S., and Godtliebsen, F., [Improved Skin Lesion Diagnostics for General Practice by Computer-Aided Diagnostics] , 1st ed., in Dermoscopy Image Anal., 1st ed., M. E. Celebi, T. Mendonca, and J. S. Marques, Eds., CRC Press, 247-292 (2015).

[26] Abedini, M., Chen, Q., Codella, N., Garnavi, R., and Sun, X., [Accurate and Scalable System for Automatic Detection of Malignant Melanoma] , 1st ed., in Dermoscopy Image Anal., 1st ed., M. E. Celebi, T. Mendonca, and J. S. Marques, Eds., CRC Press, 293-343 (2015).

[27] Schuh, S., Kaestle, R., Sattler, E., and Welzel, J., "Comparison of different optical coherence tomography devices for diagnosis of non-melanoma skin cancer," Skin Research and Technology 22(4), 395-405 (2016).

[28] "VivoSight Scanner - Vivosight," Michelson Diagnostics Ltd, <https://vivosight.com/patient/vivosight-scanner/> (10 December 2018).

[29] Dermalumics, "NITID device," [No longer available], <http://www.dermalumics.com/nitid/> (21 July 2018).

[30] Thorlabs, "Callisto 930 nm OCT Imaging System,"

$<$ https://www.thorlabs.com/newgrouppage9.cfm?objectgroup_id=3779> (10 December 2018).

[31] "MedLumics > MedLumics Introduces NITID Skin Imaging System at EADV," $<$ https://www.medlumics.com/medlumics-presenta-nitid-skin-imaging-system-en-la-eadv/> (19 January 2019).

[32] Galdran, A., Alvarez-Gila, A., Meyer, M.I., Saratxaga, C.L., Araújo, T., Garrote, E., Aresta, G., Costa, P., Mendonça, A.M., et al., "Data-Driven Color Augmentation Techniques for Deep Skin Image Analysis," in ISIC 2017 Chall. (2017).

[33] Otsu, N., "A Threshold Selection Method from Gray-Level Histograms," IEEE Transactions on Systems, Man, and Cybernetics 9(1), 62-66 (1979).

[34] Gonzalez, R.C., and Woods, R.E., [Digital Image Processing (3rd Edition)] , Prentice-Hall, Inc., Upper Saddle River, NJ, USA (2006). 
[35] Lee, T., Ng, V., Gallagher, R., Coldman, A., and McLean, D., "Dullrazor ${ }^{\circledR}$ : A software approach to hair removal from images," Computers in Biology and Medicine 27(6), 533-543 (1997).

[36] Abbas, Q., Garcia, I.F., Emre Celebi, M., and Ahmad, W., "A Feature-Preserving Hair Removal Algorithm for Dermoscopy Images,” Skin Research and Technology 19(1), e27-e36 (2013).

[37] De Carvalho, N., Schuh, S., Kindermann, N., Kästle, R., Holmes, J., and Welzel, J., "Optical coherence tomography for margin definition of basal cell carcinoma before micrographic surgery - recommendations regarding the marking and scanning technique," Skin Research and Technology 24(1), 145-151 (2018).

[38] Welzel, J., and Schuh, S., "OCT margin mapping: The training guide for Mohs surgeons - YouTube," OCT Focus, 2018, <https://www.youtube.com/watch?v=CUUanxi-LdM> (12 June 2018).

[39] Asaoka, R., Murata, H., Hirasawa, K., Fujino, Y., Matsuura, M., Miki, A., Kanamoto, T., Ikeda, Y., Mori, K., et al., "Using Deep Learning and transform learning to accurately diagnose early-onset glaucoma from macular optical coherence tomography images.," American journal of ophthalmology (2018).

[40] De Fauw, J., Ledsam, J.R., Romera-Paredes, B., Nikolov, S., Tomasev, N., Blackwell, S., Askham, H., Glorot, X., O’Donoghue, B., et al., "Clinically applicable deep learning for diagnosis and referral in retinal disease," Nature Medicine 24(9), 1342-1350 (2018). 